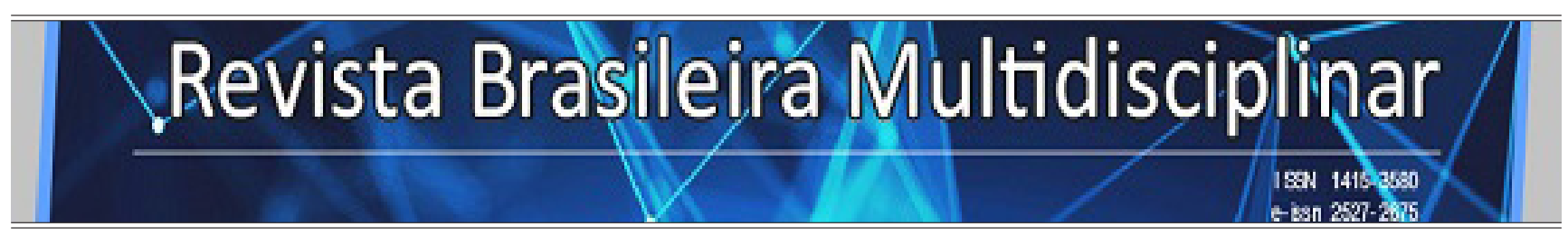

http://revistarebram.com/index.php/revistauniara

\title{
DISFUNÇÃo TEMPOROMANDIBULAR EM ADOLESCENTES E SUA RELAÇÃo COM HÁBITOS PARAFUNCIONAIS
}

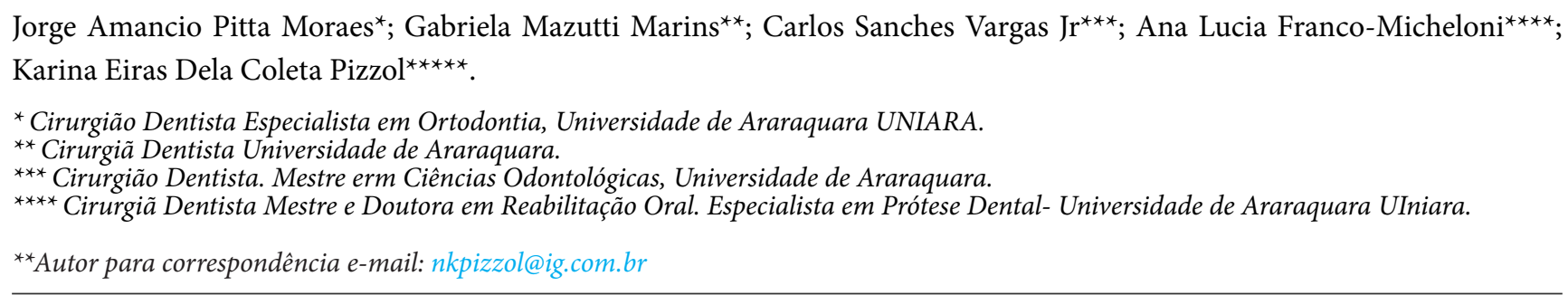

\section{Palavras-Chave}

Síndrome da Disfunção da Articulação Temporomandibular

Hábitos

Adolescente

\section{KEYWORDS}

Temporomandibular Joint Dysfunction Syndrome

Habits

Adolescents
RESUMO: Disfunção temporomandibular (DTM) é um termo que engloba uma série de problemas que afetam a articulação temporomandibular (ATM) e/ou os músculos da mastigação e estruturas associadas. A DTM pode manifestar-se na forma de dor na face e/ou região das ATM, comprometendo significativamente a qualidade de vida do seu portador. Fundamentada na teoria multifatorial da DTM, a literatura evidencia como fatores causais: trauma, hábitos parafuncionais, fatores estruturais, psicológicos e posturais. Embora a DTM possa afetar pacientes de todas as faixas etárias e de ambos os gêneros, seu mecanismo de ação e sua evolução são pouco explorados na fase da adolescência. Assim, este trabalho se propõe a realizar uma revisão da literatura sobre a DTM e sua relação com hábitos parafuncionais em pacientes adolescentes, abrangendo os sinais e sintomas mais prevalentes e o gênero mais atingido por essas disfunções. Foram revisados 67 artigos científicos e livros, utilizando as bases de dados PubMed, LILACs, MEDLINE, ScieELO e Google Acadêmico e as palavraschave Síndrome da Disfunção da Articulação Temporomandibular; Hábitos e Adolescente; e seus respectivos termos em inglês. De acordo com os artigos revisados, pôde-se concluir que a DTM possui elevada prevalência na população jovem, principalmente no gênero feminino, embora sua fisiopatologia nessa faixa etária ainda seja pouco estudada. Além disso, hábitos parafuncionais, fatores estruturais, psicológicos e posturais podem aumentar a probabilidade de desenvolver sinais e sintomas de DTM.

\begin{abstract}
TEMPOROMANDIBULAR DYSFUNCTION IN ADOLESCENTS AND ITS RELATIONSHIP WITH PARAFUNCTIONAL HABITS

temporomandibular disorder (TMD) is a term that encompasses a number of disorders that affect the temporomandibular joint (TMJ) and / or the masticatory muscles and associated structures. The DTM can manifest itself in the form of pain in the face and / or the TMJ region, significantly affecting the quality of life of its bearer. Based on the multifactorial TMD theory, the literature shows as causal factors: trauma, parafunctional habits, structural, psychological and postural factors. Although the DTM can affect patients of all age groups and both genders, its mechanism of action and its evolution is little known in adolescence. This work intends to perform a literature review on temporomandibular joint dysfunction and its relationship with parafunctional habits of adolescents, including the signs and symptoms most prevalent and the most affected by these disorders gender. We reviewed 67 papers and books, using the PubMed, Lilacs, MEDLINE, ScieELO and Google Scholar and keywords Syndrome Temporomandibular Joint Dysfunction; Habits Adolescents; and their respective terms in English. According to the revised articles, we concluded that the DTM has a high prevalence in young people, especially in females, though its pathophysiology is poorly studied; and parafunctional factors, structural, psychological and postural may increase the likelihood of developing signs and symptoms of TMD.
\end{abstract}




\section{INTRODUÇÃo}

O sistema estomatognático é composto pela mandíbula, maxila, arcadas dentárias, tecidos moles, articulação temporomandibular (ATM) e músculos. Quando ocorre um desequilíbrio desses componentes, pode ocorrer o desenvolvimento de disfunções temporomandibulares (DTM), descritas como um termo que engloba uma série de problemas clínicos que afetam a ATM e/ ou os músculos da mastigação e estruturas associadas (OKESON, 2013). A DTM pode manifestar-se na forma de dor na face, cabeça e/ ou região das ATM, comprometendo significativamente a qualidade de vida do seu portador (MAIXNER et al., 2011). Outros sintomas comuns são ruídos articulares, cansaço muscular, além de movimentos irregulares ou limitados da mandíbula (PEREIRA et al., 2009; ORAL et al.,2009; KITSOULIS et al., 2011; OKESON, 2013; FRANCO-MICHELONI et al., 2015; SILVA et al., 2016; BENOLIEL et al., 2017; CHATZOPOULOS et al., 2017; BERTOLI et al.,2018; FERNANDES et al., 2019; DE MELO JR et al., 2019).

A etiologia da DTM é considerada multifatorial (ORAL et al., 2009; FERNADES et al., 2015) e inclui fatores predisponentes, fatores desencadeantes e fatores que mantêm a dor (perpetuantes). Os fatores de risco para o início da DTM em adolescentes são similares àqueles para o início de outras condições de dor, assim como para o aparecimento de DTM e outras dores em adultos (ORAL et al., 2009; ORTEGA e GUIMARÃES, 2013). Pressupõe-se desta forma que, fatores oclusais associados às respostas nos músculos da mastigação e ATM e às condições gerais e emocionais do paciente podem iniciar, manter ou agravar o quadro (PEREIRA et al., 2009; KITSOULIS et al., 2011). Fundamentada na teoria multifatorial, a literatura aponta como possíveis fatores causais traumas na região de cabeça e pescoço, parafunção, fatores psicológicos e má oclusão (ORAL et al., 2009; MAIXNER et al., 2011).

Fatores mecânicos locais, como os hábitos parafuncionais, apresentam papel importante na etiologia da dor orofacial, podendo sua influência variar segundo a tolerância do paciente à dor e suas diferentes respostas bioquímicas e fisiológicas a esses fatores. Denominam-se hábitos parafuncionais aqueles não relacionados à execução das funções normais do sistema estomatognático, como a deglutição, mastigação e fonação (OKESON, 2013). Estudos envolvendo adolescentes, como o realizado por MOTTA et al. (2013), demonstraram significativa associação entre sinais e sintomas de DTM e presença de hábitos parafuncionais por via oral, como: roer as unhas, morder objetos/lábios e ranger ou apertar os dentes (FERNANDES et al., 2015; FRANCO-MICHELONI et al., 2015). Por sua vez, REZENDE et al. (2009), consideram ainda que o gênero feminino apresenta o dobro da prevalência para hábitos parafuncionais em relação ao masculino, um dos fatores que poderia favorecer os índices elevados de DTM nesse gênero, conforme observado na literatura (FARSI 2003; FEITH RABAB, 2006; GOYATÁ et al., 2010; EBRAHIMI et al., 2011; KHAN et al., 2020).

De forma geral, a prevalência de DTM em adultos é considerada elevada, variando entre 21,5\% e 51,8\% (GESCH et al., 2004; GONÇALVES et al., 2010, MAIXNER et al., 2011). Em contrapartida, os escassos trabalhos envolvendo crianças e adolescentes reportam prevalências variáveis (FRANCO-MICHELONI et al., 2015; RUBIN et al., 2017; BERTOLI et al., 2018; MARPAUNG, LOBBEZZO e VAN SELMS, 2018). Embora a DTM possa afetar pacientes de todas as faixas etárias e de ambos os gêneros, seu mecanismo de ação e sua evolução são pouco explorados na fase da adolescência e os fatores de risco envolvidos ainda são controversos (BONJARDIM et al., 2005; BARBOSA et al., 2010; MAIXNER et al., 2011; FRANCO-MICHELONI et al., 2015; CHATZOPOULOS et al.; 2017; BERTOLI et al., 2018; DE MELO JR et al., 2019). Fatores emocionais, hormonais e hábitos parafuncionais frequentes nessa faixa etária, como mascar chiclete, morder objeto, onicofagia, e também o bruxismo estão normalmente envolvidos com o surgimento da DTM (FERNANDES et al., 2015; FRANCO-MICHELONI et al., 2015; CHATZOPOULOS et al.; 2017). Uma vez que os sinais e sintomas de DTM podem se iniciar na adolescência e perdurar até a idade adulta (CARLSSON, EGERMARK e MAGNUSSON, 2002; NILSSON e LIST, 2020), é interessante investigar sua prevalência e fatores associados nessa faixa etária. 
Assim, este artigo teve como Oobjetivo realizar uma revisão da literatura sobre a DTM e sua relação com hábitos parafuncionais em adolescentes, abrangendo os sinais e sintomas mais prevalentes e o gênero mais atingido por essas disfunções.

\section{MATERIAL E MÉTODOS}

A revisão de literatura foi realizada a partir da busca exploratória, nas bases de dados Pubmed, Scielo, LILACS e Google Acadêmico. Foram selecionados diversos textos científicos, publicados nos últimos 20 anos (2001-2020), sobre hábitos parafuncionais e DTM na adolescência, sendo excluídos da seleção artigos de relatos de casos clínicos. Foram empregados os descritores "síndrome da Disfunção da articulação temporomandibular", "hábitos" e "adolescente", bem como os termos equivalentes em inglês, "temporomandibular joint dysfunction syndrome" "habits" e "adolescents". Ao todo, foram selecionados 67 artigos, livros e teses que atendiam aos critérios descritos, sendo 12 nacionais e 55 internacionais.

\section{ConTeúdo}

Estima-se que mais de 75\% da população adulta apresente algum sinal ou sintoma de DTM, como a disfunção miofascial. Entretanto, a prevalência de DTM descrita na literatura é menos significativa, variando entre $21,5 \%$ e 51,8\%, sendo mais prevalente entre as mulheres (GESCH et al., 2004; GONÇALVES et al., 2010, MAIXNER et al., 2011). Na faixa etária entre 10 e 19 anos, que delimita a adolescência, estima-se que a prevalência de DTM e sinais associados seja de 20\% a 85\% (FRANCO-MICHELONI et al., 2015; SPALJ et al., 2015; ARAVENA et al., 2016; OLIVEIRA et al., 2016; AGARWAL, SAHA e SINHA, 2016; KHAN, GORNITSKY e VELLY, 2016; ANITHA e KUMAR, 2017; RUBIN et al., 2017; BERTOLI et al., 2018; BARRETO et al., 2018; MARPAUNG; LOBBEZZO; VAN SELMS, 2018), podendo sofrer variações de acordo com fatores como gênero, aumento da idade, fatores emocionais (estresse), qualidade e horas de sono (SENA et al., 2013; KIM, RYU e AHN, 2015; BENOLIEL et al., 2017; MARPAUNG; LOBBEZZO; VAN SELMS, 2018; KHAN et al., 2020).

Diante de números tão contrastantes, considera-se que a prevalência de DTM em crianças e adolescentes seja difícil de estabelecer. Muitos pesquisadores acreditam que é necessário ter uma classificação padronizada para avaliar os sinais e sintomas da DTM, para medir e comparar a gravidade dos distúrbios da ATM e avaliar a condição do paciente após o tratamento, enfatizando a necessidade de uma ferramenta eficaz para estudar os fatores etiológicos (KHOJASTEPOUR; VOJDANI; FORGHANI, 2017), permitindo assim, números mais confiáveis relacionados à DTM nessa faixa etária. Algumas dessas ferramentas já foram testadas e validadas para pesquisas científicas com adolescentes, como o Research Diagnostic Criteria for Temporomandibular Disorders (RDC/TMD), também com versão em português (PEREIRA-JÚNIOR; FAVILLA; DWORKIN, 2004). Segundo a literatura, o Eixo I do RDC/TMD pode ser aplicado tanto em adultos quanto em adolescentes (PEREIRA-JÚNIOR; FAVILLA; DWORKIN, 2004; FRANCO-MICHELONI et al., 2015; CAMPI et al., 2020). Embora a fisiopatologia da DTM não seja ainda totalmente elucidada, seus sinais e sintomas são normalmente clássicos. Dores musculares, limitação e desvio na trajetória mandibular, zumbidos no ouvido, dores e ruídos articulares durante a abertura e fechamento bucal, artralgia, deslocamento do disco, dores na cabeça, nuca e/ou pescoço e dores de ouvido, dores ou sensibilidade nos dentes e cansaço durante a mastigação são alguns dos sinais e sintomas mais relatados (TOSATO; CARIA, 2006; FEITH RABAB, 2006; AREBALO et al., 2010; FRANCO-MICHELONI et al., 2015; KARIBE et al., 2015; MOTGHARE et al., 2015; MEJERSJÖ; OVESSON; MOSSBERG, 2015; SILVA et al., 2016; ARAVENA et al., 2016; KHAN; GORNITSKY; VELLY, 2016; BENOLIEL et al., 2017; CHATZOPOULOS et al., 2017; RIBEIRO-DASILVA; FILLINGIM; WALLET, 2017; BERTOLI et al.,2018).

$\mathrm{Na}$ adolescência, é possível que haja um aumento na incidência de condições dolorosas vivenciadas por ser uma fase de grandes mudanças hormonais e um período de alterações culturais e comportamentais 
(RIBEIRO-DASILVA; FILLINGI; WALLET, 2017; FRANCO-MICHELONI et al., 2015). Durante a primeira década de vida, a criança apresenta dificuldade em verbalizar a localização precisa e a natureza da dor facial e disfunção mandibular que muitas vezes resulta em uma história não definitiva, aumentando a importância da percepção do dentista quanto aos primeiros sinais e sintomas de distúrbios da ATM (NILSON; LIST; DRANGSHOLT, 2006; NILSON, LIST e DRANGSHOLT, 2007; HOWARD, 2013). A partir da adolescência, o paciente apresenta melhora na capacidade de percepção e expressão da dor, o que facilita o diagnóstico da DTM (NILSON; LIST; DRANGSHOLT, 2006; NILSON; LIST; DRANGSHOLT, 2007; NILSSON; WILLMAN, 2016).

De forma geral, os sinais e sintomas dolorosos de DTM na adolescência costumam apresentar maior envolvimento muscular do que articular (NILSON; LIST; DRANGSHOLT, 2006; FERNANDES et al., 2016), salvo as exceções de quadros infecciosos sistêmicos, como artrite reumatóide juvenil. Por outro lado, autores como HIRSCH; HOFFMANN; TÜRP (2012) e VIEROLA et al. (2012) apontaram os sons articulares como um dos sintomas mais frequentes de DTM em adolescentes. Nesta fase, as ATMs possuem grande potencial de crescimento, além de elevada capacidade adaptativa e remodelatória, o que faz com que os danos articulares não ocorram com tanta frequência. Em contrapartida, o elevado tônus muscular e força de mordida molar máxima presente nesta faixa etária, faz com que a hipertonia e fadiga muscular sejam uma constante na DTM juvenil. Vale ressaltar que essas condições de dor estão associadas à considerável morbidade, custos sociais e redução da qualidade de vida dos indivíduos acometidos (SILVA et al., 2016; RIBEIRO-DASILVA et., 2017).

A etiologia é diversificada, por isso dizemos que a DTM é de origem multifatorial (ORAL et al., 2009; FERNANDES et al., 2015). Dentre as possíveis causas estão a hiperatividade muscular, trauma, estresse emocional, má oclusão, problemas esqueléticos/posturais, fatores psicológicos e hábitos parafuncionais. Tais fatores associados podem causar, manter ou agravar a DTM, além de promover luxações ou subluxações da ATM e deslocamento do disco articular (BRANCO et al., 2008). Segundo STEIN et al. (2017) não há padrão esquelético ou classe dentária relacionada a graus mais elevados de desarranjo interno nas ATMs de crianças e adolescentes que apresentam sinais clínicos de DTM. Portanto, não é possível tirar conclusões sobre a gravidade do desarranjo interno em relação à classe dentária e esquelética em ATMs juvenis sintomáticas.

O fato da DTM ser de caráter multifatorial, significa que os indivíduos podem apresentar sintomas semelhantes, mas com causas completamente diferentes (FRANCO-MICHELONI et al., 2015). O controle da dor é sabidamente influenciado por vários fatores, como origem, variação, duração, efeito placebo e a habilidade do terapeuta em colocar em prática o tratamento sugerido. Portanto, é importante que se diferencie clinicamente o perfil dos pacientes, por meio da anamnese, a fim de individualizar o tratamento.

A relação entre DTM e hábitos parafuncionais é atualmente descrita na literatura (PEREIRA et al., 2010; ARAÚJO; COELHO; GUIMARÃES, 2011; CARDOSO; KRAYCHETE; ARAÚJO, 2011; MOTTA et al., 2013; FRANCO-MICHELONI et al., 2015; KIM; RYU; AHN, 2015; MEJERSJÖ; OVESSON; MOSSBERG, 2015; MOTGHARE et al., 2015; SPALJ et al., 2015; AGARWAL; SAHAH; SINHA, 2016; ARAVENA et al., 2016; FERNANDES et al., 2016; OLIVEIRA et al., 2016; SILVA et al., 2016; ANITHA; KUMAR, 2017; BERNOLIEL et al., 2017; RUBIN et al., 2017; BARRETO et al., 2018; BERTOLI et al., 2018; MARPAUNG; LOBBEZOO; VAN SELMS, 2018; PERROTTA et al., 2019; SARIT et al., 2019; TANEJA et al., 2019; NILSSON, LIST, 2020). Denominam-se, genericamente, hábitos parafuncionais os hábitos orais nocivos realizados sem a consciência do indivíduo (OKESON, 2013). Atualmente, o bruxismo do sono e o apertamento diurno são considerados entidades distintas das parafunções por apresentarem fisiopatologia diferente (LOBBEZOO et al., 2013). Quando excedem o limite de tolerância fisiológica do individuo, essas atividades acarretam uma agressão ao sistema estomatognático (REZENDE et al., 2009). Essas atividades geram sensibilização periférica e podem levar a dores agudas locais nos tecidos 
acometidos pela sobrecarga funcional. A sensibilização periférica quando não resolvida (pela ausência de tratamento efetivo), pode então tornar-se uma fonte de dor contínua no tecido que leva à sensibilização central (OHRBACH et al., 2011; FERNANDES et al., 2012; OKESON, 2013;CAMPI et al., 2020). Assim, o estresse mecânico gerado por esses hábitos favorece a fratura e/ou desgaste de dentes e restaurações, danos à estrutura periodontal de suporte, ou mesmo, exacerbação de DTM e outras dores orofaciais. De modo geral, a busca por tratamento só ocorre após o aparecimento dos danos e, principalmente, por queixa de dor. É essencial que o diagnóstico da DTM ocorra na sua fase inicial, uma vez que alguns danos provocados podem ser irreversíveis. Pacientes jovens respondem bem a modalidades conservadoras de tratamento que incluem medidas protetoras (ex: placas oclusais rígidas) ou minimizadoras (ex: educação, autocuidados) capazes de preservar a integridade das estruturas bucais e a saúde da ATM e dos músculos da mastigação (WAHLUND; NILSSON; LARSSON, 2015).

As pesquisas sobre DTM e hábitos parafuncionais em amostras de pacientes jovens são relativamente recentes e sua importância é relevante uma vez que os hábitos orais nocivos podem iniciar na infância e perdurarem até a idade adulta e, uma vez incorporados no subconsciente, os indivíduos não os percebem quando fazem (ORTEGA; GUIMARÃES, 2013). Algumas das principais pesquisas publicadas nos últimos anos estão descritas no Quadro 1. Poucas são as que descrevem de forma precisa e com evidências científicas as principais causas, sinais/sintomas, progressão da disfunção e terapias mais efetivas nessa faixa etária. De acordo com os artigos revisados envolvendo pacientes jovens, a prevalência de sinais e sintomas de DTM variou entre 20,7 e $84,5 \%$ enquanto que a frequência de hábitos parafuncionais foi ainda maior (47,5 a 93\%) (FARSI 2003; CAUÁS et al., 2004; FEITH RABAB, 2006; BRANCO et al., 2008; FRAGOSO et al., 2010; GOYATÁ et al., 2010; EBRAHIMI et al., 2011; MEJERSJÖ; OVESSON; MOSSBERG, 2015; OLIVEIRA et al., 2016; ANITHA; KUMAR, 2017; RUBIN et al., 2017; ATSÜ et al., 2019), havendo uma predominância clara do gênero feminino sobre o masculino, podendo chegar ao dobro da sua prevalência (FARSI 2003; FEITH RABAB, 2006; GOYATÁ et al., 2010; EBRAHIMI et al., 2011; FRANCO-MICHELONI et al., 2015; MEJERSJÖ; OVESSON; MOSSBERG, 2015; SPALJ et al., 2015; ARAVENA et al., 2016; KHAN; GORNITSKY; VELLY, 2016; OLIVEIRA et al., 2016; BERTOLI et al., 2018; MARPAUNG; LOBBEZOO; VAN SELMS, 2018; CAMPI et al., 2020).

Diante desse contexto, PEREIRA et al. (2010) propuseram verificar a associação entre gênero, presença de menarca, oclusão, comportamento gestacional da mãe e hábitos orais como indicadores de risco para DTM em crianças de 12 anos de idade. Através deste estudo, concluíram que o comportamento gestacional da mãe e a presença da menarca, má oclusão e hábitos orais da criança não foram associados com o diagnóstico de DTM e que o gênero foi o único fator associado com a incidência de DTM em crianças de 12 anos, sendo as meninas mais frequentemente afetadas. A relação entre hábitos parafuncionais e DTM, presente entre pais e filhos também não foi observada na literatura (ARAÚJO; COELHO; GUIMARÃES, 2011).

MELCHIOR; MAZZETTO; FELÍCIO (2012), avaliaram a frequência e a gravidade dos sinais e sintomas da DTM e da frequência de hábitos bucais deletérios. Analisaram também possíveis correlações entre a idade dos sujeitos, a gravidade e frequência dos sinais e sintomas, número de hábitos e duração da DTM. Com base na anamnese, exame clínico, palpação dos músculos da mastigação e das ATMs, obtiveram como resultado que a idade dos indivíduos foi positivamente relacionada com a gravidade da dor muscular, dor na ATM, dor de ouvido, dor de garganta e sensibilidade do dente. O grau da DTM foi diretamente relacionado com a gravidade da dor muscular, da dor na ATM, do zumbido e plenitude auricular. Já a frequência de hábitos foi negativamente correlacionada com a idade dos indivíduos e também não houve correlação entre o número de hábitos bucais deletérios e severidade da DTM. Concluíram assim, que a quantidade de sinais e sintomas e sua duração, podem ser indicativos da severidade da DTM. Os sintomas de bruxismo e apertamento foram significativos e os sintomas de zumbido e plenitude auricular foram correlacionados com o tempo da disfunção e não com a idade. 
Quadro 1- Resumo dos artigos mais recentes sobre DTM em adolescentes.

\begin{tabular}{|c|c|c|c|}
\hline Autor(es), ano & $\begin{array}{l}\text { Tipo de } \\
\text { estudo }\end{array}$ & Amostra & Principais achados \\
\hline NILSSON e LIST, 2020 & $\begin{array}{l}\text { Estudo } \\
\text { clínico }\end{array}$ & $\begin{array}{l}2209 \text { adolescentes } \\
\text { (sem descrição } \\
\text { de faixa etária e } \\
\text { nacionalidade) }\end{array}$ & $\begin{array}{l}\text { Indivíduos com DTM dolorosa tiveram elevada frequência } \\
\text { de dores comórbidas, } 45,2 \% \text { relataram escores de } \\
\text { depressão moderada a grave e } 13,0 \% \text { tinham incapacidade } \\
\text { moderada relacionada à dor. A DTM dolorosa na } \\
\text { adolescência parece triplicar o risco de dor relacionada à } \\
\text { DTM em adultos jovens, e a dor persistente aumenta a dor } \\
\text { comórbida e o sofrimento psicossocial. }\end{array}$ \\
\hline SARIT et al., 2019 & $\begin{array}{l}\text { Estudo } \\
\text { clínico }\end{array}$ & $\begin{array}{l}90 \text { adolescentes } \\
\text { indianos } \\
(12-15 \text { anos })\end{array}$ & $\begin{array}{l}\text { Hábitos parafuncionais, má oclusão e DTM tiveram } \\
\text { associação estatisticamente significativa com saúde oral } \\
\text { relacionada à qualidade de vida. Os hábitos parafuncionais } \\
\text { e a má oclusão foram considerados preditores } \\
\text { significativos para DTM e saúde oral relacionada à } \\
\text { qualidade de vida. As DTM e os fatores associados têm } \\
\text { impacto no funcionamento psicossocial das crianças. }\end{array}$ \\
\hline PERROTTA et al., 2019 & $\begin{array}{l}\text { Estudo } \\
\text { clínico }\end{array}$ & $\begin{array}{l}700 \text { adolescentes } \\
\text { italianos (9-11 } \\
\text { anos) }\end{array}$ & $\begin{array}{c}\text { Má oclusão, parafunções orais e dor por DTM são achados } \\
\text { frequentes entre escolares italianos e alguns fatores oclusais } \\
\text { e a alta frequência de parafunções orais podem estar } \\
\text { associados à DTM dolorosa. }\end{array}$ \\
\hline GÖRÜŞ e ÜNER, 2019 & $\begin{array}{l}\text { Estudo } \\
\text { clínico }\end{array}$ & $\begin{array}{l}63 \text { estudantes } \\
\text { universitários da } \\
\text { Turquia } \\
\text { (sem especificação } \\
\text { da idade) }\end{array}$ & $\begin{array}{l}\text { 73\% dos participantes tinham algum grau DTM. } \\
\text { Concluíram que o aumento dos hábitos parafuncionais } \\
\text { também promoveu aumento na prevalência de distúrbio } \\
\text { da ATM. }\end{array}$ \\
\hline TANEJA et al., 2019 & $\begin{array}{l}\text { Estudo } \\
\text { clínico }\end{array}$ & $\begin{array}{l}300 \text { adolescentes } \\
\text { indianos } \\
(12-15 \text { anos })\end{array}$ & $\begin{array}{l}\text { A prevalência geral de sinais e sintomas de DTM foi de } \\
51 \% \text {. O gênero feminino apresentou maior prevalência } \\
(57,1 \%) \text { de sintomas de DTM quando comparado ao } \\
\text { gênero masculino }(43,2 \%) \text {. Os sinais de DTM mostraram } \\
\text { relação estatisticamente significativa com sobremordida } \\
\text { (p=0,007) e bruxismo (p<0,0001). Nenhuma relação } \\
\text { significativa foi encontrada entre as más oclusões e sinais } \\
\text { de DTM, exceto para sobremordida e bruxismo, mas uma } \\
\text { relação significativa foi encontrada entre os sintomas de } \\
\text { DTM e a má oclusão. }\end{array}$ \\
\hline $\begin{array}{l}\text { MARPAUNG, VAN } \\
\text { SELMS, e LOBBEZOO, } \\
2018\end{array}$ & $\begin{array}{l}\text { Estudo } \\
\text { clínico }\end{array}$ & $\begin{array}{l}1800 \text { crianças } \\
\text { e adolescentes } \\
\text { indonésias } \\
(7-18 \text { anos })\end{array}$ & $\begin{array}{l}\text { Fatores psicológicos e presença de dor corporal estiveram } \\
\text { fortemente associados às DTM, ao lado dos hábitos } \\
\text { orais (em crianças) e bruxismo do sono e vigília (em } \\
\text { adolescentes) sendo portanto, considerados indicadores de } \\
\text { risco para DTM dolorosa. }\end{array}$ \\
\hline BARRETO et al., 2018 & $\begin{array}{l}\text { Estudo } \\
\text { clínico }\end{array}$ & $\begin{array}{l}167 \text { adolescentes } \\
\text { universitários } \\
\text { colombianos } \\
\text { (sem especificação } \\
\text { da idade) }\end{array}$ & $\begin{array}{c}\text { 40\% da amostra apresentou DTM; observou-se alta } \\
\text { presença de hábitos orais parafuncionais associados à } \\
\text { DTM. }\end{array}$ \\
\hline BERTOLI et al., 2018 & $\begin{array}{l}\text { Estudo } \\
\text { clínico }\end{array}$ & $\begin{array}{l}934 \text { adolescentes } \\
\text { brasileiros } \\
(10-14 \text { anos })\end{array}$ & $\begin{array}{l}\text { Sintomas de DTM estavam presentes em } 34,9 \% \text { da amostra, } \\
\text { sendo a dor miofascial o tipo mais prevalente (10,3\%); } \\
\text { maior prevalência em meninas. }\end{array}$ \\
\hline $\begin{array}{l}\text { CHATZOPOULOS et } \\
\text { al., } 2017\end{array}$ & $\begin{array}{l}\text { Estudo } \\
\text { clínico }\end{array}$ & $\begin{array}{l}4204 \text { adolescentes } \\
\text { americanos }(11-15 \\
\text { anos) }\end{array}$ & $\begin{array}{l}\text { Bruxismo e apertamento foram relatados por } 26,5 \% \text { da } \\
\text { população examinada; } 14,8 \% \text { apresentaram estalidos } \\
\text { e 3,6\% dificuldade de mastigação e fechamento. Idade } \\
\text { e gênero associaram-se aos parâmetros examinados (p } \\
<0,05) .\end{array}$ \\
\hline $\begin{array}{c}\text { ANITHA e KUMAR, } \\
2017\end{array}$ & $\begin{array}{l}\text { Estudo } \\
\text { clínico }\end{array}$ & $\begin{array}{l}100 \text { adolescentes } \\
\text { indianos (sem } \\
\text { especificação da } \\
\text { idade) }\end{array}$ & $\begin{array}{c}\text { Sessenta participantes tinham pelo menos sintomas } \\
\text { subclínicos leves de DTM e } 75 \% \text { da amostra pelo menos } \\
\text { um hábito parafuncional. }\end{array}$ \\
\hline
\end{tabular}


Quadro 1- Resumo dos artigos mais recentes sobre DTM em adolescentes (cont.).

\begin{tabular}{|c|c|c|c|}
\hline RUBIN et al., 2017 & $\begin{array}{l}\text { Estudo } \\
\text { clínico }\end{array}$ & $\begin{array}{l}153 \text { crianças e } \\
\text { adolescentes da } \\
\text { Uganda (6-17 } \\
\text { anos) }\end{array}$ & $\begin{array}{c}\text { Prevalência moderada (35\%) de DTMs na amostra; } \\
\text { Hábitos parafuncionais relatados por 93\% dos } \\
\text { participantes e significativamente relacionados à mialgia. } \\
\text { Não foram encontradas diferenças significativas quanto ao } \\
\text { gênero ou idade. Sono e bruxismo não foram relacionados } \\
\text { à DTM. }\end{array}$ \\
\hline BENOLIEL et al.,2017 & $\begin{array}{l}\text { Estudo } \\
\text { clínico }\end{array}$ & $\begin{array}{l}286 \text { jovens (sem } \\
\text { especificação } \\
\text { de idade e } \\
\text { nacionalidade) }\end{array}$ & $\begin{array}{c}\text { Os pacientes com DTM apresentaram pior qualidade } \\
\text { de sono, estando o sono, positivamente associado às } \\
\text { características da DTM, comorbidades e hábito de } \\
\text { apertamento. }\end{array}$ \\
\hline$\frac{\text { AGARWAL, SAHA }}{\text { SINHA, } 2016}$ & $\begin{array}{l}\text { Estudo } \\
\text { clínico }\end{array}$ & $\begin{array}{l}407 \text { adolescentes } \\
\text { indianos }(15-17 \\
\text { anos })\end{array}$ & $\begin{array}{l}\text { A prevalência de DTM foi de } 22,4 \% \text {, não havendo } \\
\text { associação entre idade, gênero e DTM. Hábitos } \\
\text { parafuncionais apresentaram associação estatisticamente } \\
\text { significante com DTM. }\end{array}$ \\
\hline SILVA et al.,2016 & $\begin{array}{l}\text { Estudo } \\
\text { clínico }\end{array}$ & $\begin{array}{l}248 \text { adolescentes } \\
\text { brasileiros (12 } \\
\text { anos) }\end{array}$ & $\begin{array}{l}\text { Os sintomas da DTM foram associados à dor e ao } \\
\text { apertamento dos dentes, causando um impacto negativo } \\
\text { na qualidade de vida dos escolares. }\end{array}$ \\
\hline $\begin{array}{l}\text { MARPAUNG et } \\
\text { al.,2016 }\end{array}$ & $\begin{array}{l}\text { Estudo } \\
\text { clínico }\end{array}$ & $\begin{array}{l}\text { 4.235adolescentes } \\
\text { holandeses }(12-18 \\
\text { anos) }\end{array}$ & $\begin{array}{l}\text { A prevalência de DTM dolorosa foi de } 21,6 \% \text { e de ruídos } \\
15,5 \% \text {. Análises de regressão logística revelaram que o } \\
\text { gênero, o aumento da idade e as parafunções mostraram } \\
\text { ser fatores predisponentes fortes de dor na DTM.. }\end{array}$ \\
\hline KHAN, 2016 & $\begin{array}{l}\text { Estudo } \\
\text { clínico }\end{array}$ & $\begin{array}{l}313 \text { adolescentes } \\
\text { canadenses }(14-17 \\
\text { anos) }\end{array}$ & $\begin{array}{l}\text { Prevalência de } 23 \% \text { de DTM dolorosa sendo as dores de } \\
\text { cabeça fortemente associadas à DTM dolorosa. Meninas } \\
\text { com DTM dolorosa apresentaram maior chance de cefaleia } \\
\text { e dor nas costas enquanto os meninos maior probabilidade } \\
\text { de cervicalgia. }\end{array}$ \\
\hline ARAVENA et al., 2016 & $\begin{array}{l}\text { Estudo } \\
\text { clínico }\end{array}$ & $\begin{array}{l}186 \text { adolescentes } \\
\text { chilenos } \\
(15,4 \pm 1,25 \text { anos })\end{array}$ & $\begin{array}{l}40 \% \text { (na maioria meninas) manifestaram algum dor } \\
\text { ou desconforto temporomandibular. Dor de cabeça, } \\
\text { apertamento os dentes, e ruídos articulares foram } \\
\text { comumente relatados. DTM muscular estava presente em } \\
10,8 \% \text { e } 11,3 \% \text { tinham alterações articulares. }\end{array}$ \\
\hline OLIVEIRA et al., 2016 & $\begin{array}{l}\text { Estudo } \\
\text { clínico }\end{array}$ & $\begin{array}{l}129 \text { adolescentes } \\
\text { brasileiros } \\
(16-19 \text { anos })\end{array}$ & $\begin{array}{l}\text { Houve uma alta incidência de sinais e sintomas de DTM } \\
(84,5 \%) \text { e hábitos parafuncionais em estudantes do ensino } \\
\text { médio, estando os mesmos associados. }\end{array}$ \\
\hline $\begin{array}{l}\text { FERNANDES et al., } \\
2016\end{array}$ & $\begin{array}{l}\text { Estudo } \\
\text { clínico }\end{array}$ & $\begin{array}{l}1094 \text { adolescentes } \\
\text { brasileiros } \\
(12-14 \text { anos })\end{array}$ & $\begin{array}{l}\text { A presença de atividades musculares concomitantes } \\
\text { durante o sono (bruxismo), apertamento diurno e outros } \\
\text { hábitos parafuncionais aumentam a probabilidade quase } \\
\text { linearmente dos adolescentes apresentarem DTM dolorosa. }\end{array}$ \\
\hline $\begin{array}{l}\text { FRANCO-MICHELONI } \\
\quad \text { et al., } 2015\end{array}$ & $\begin{array}{l}\text { Estudo } \\
\text { clínico }\end{array}$ & $\begin{array}{l}1307 \text { adolescentes } \\
\text { brasileiros }(12-14 \\
\text { anos })\end{array}$ & $\begin{array}{l}\text { 30,4\% dos adolescentes apresentaram DTM, dos quais } \\
\text { 25,2\% tinham dor. Alguns fatores mostraram-se associados } \\
\text { ao diagnóstico de DTM: queixas de cefaléia, parafunções, } \\
\text { bruxismo, apertamento diurno e pais que não viviam } \\
\text { juntos. }\end{array}$ \\
\hline MEJERSJÖ et al.,2015 & $\begin{array}{l}\text { Estudo } \\
\text { clínico }\end{array}$ & $\begin{array}{l}124 \text { adolescentes } \\
\text { suecos do ensino } \\
\text { médio (sem } \\
\text { especificação da } \\
\text { idade) }\end{array}$ & $\begin{array}{l}\text { Concluíram que existe associação entre o uso de goma de } \\
\text { mascar, roer unhas, piercing oral e sintomas de DTM. }\end{array}$ \\
\hline SPALJ et al.,2015 & $\begin{array}{l}\text { Estudo } \\
\text { clínico }\end{array}$ & $\begin{array}{l}1597 \text { adolescentes } \\
\text { croatas }(11-19 \\
\text { anos })\end{array}$ & $\begin{array}{l}22 \% \text { dos adolescentes apresentaram sinais de DTM. } \\
\text { Idade, gênero feminino e hábitos parafuncionais foram } \\
\text { relacionados a varios sinais de DTM. }\end{array}$ \\
\hline
\end{tabular}


Quadro 1- Resumo dos artigos mais recentes sobre DTM em adolescentes (cont.).

\begin{tabular}{|c|c|c|c|}
\hline KIM, RYU e AHN, 2015 & $\begin{array}{c}\text { Estudo } \\
\text { clínico }\end{array}$ & $\begin{array}{c}522 \text { adolescentes } \\
\text { coreanos (11-19 } \\
\text { anos) }\end{array}$ & $\begin{array}{c}\text { Gênero e idade influenciaram significativamente a taxa } \\
\text { de prevalência de DTM e as horas de sono. As queixas } \\
\text { principais, os hábitos parafuncionais, os diagnósticos } \\
\text { (artralgia, mialgia), dores de cabeça/pescoço/ombro e } \\
\text { tratamento não mostraram relação significativa com as } \\
\text { horas de sono. Concluiram que com o aumento da idade } \\
\text { em adolescentes, a taxa de prevalência de DTM aumentou } \\
\text { e as horas de sono diminuíram }\end{array}$ \\
\hline $\begin{array}{c}\text { MOTGHARE et al., } \\
2015\end{array}$ & $\begin{array}{c}\text { Estudo } \\
\text { clínico }\end{array}$ & $\begin{array}{c}240 \text { adolescentes } \\
\text { indianos }(10-19 \\
\text { anos) }\end{array}$ & $\begin{array}{c}\text { Dor de cabeça, dor cervical e dor de dente foram os sinais } \\
\text { e sintomas mais frequentes de DTM (46,2\%). Houve } \\
\text { associação entre gênero e DTM e entre morder a unha, } \\
\text { morder objetos e apertamento com sinais e sintomas de } \\
\text { DTM. }\end{array}$ \\
\hline KARIBE et al., 2015 & $\begin{array}{c}\text { Estudo } \\
\text { clínico }\end{array}$ & $\begin{array}{c}1415 \text { adolescentes } \\
\text { japonesas } \\
(11-15 \text { anos) }\end{array}$ & $\begin{array}{c}\text { Os sintomas de DTM estavam associados a outras } \\
\text { condições de dor orofacial, particularmente dor no } \\
\text { pescoço e cefaléia. O apertamento diurno foi fortemente } \\
\text { associado com sintomas de DTM. }\end{array}$ \\
\hline FERNANDES et al., & $\begin{array}{c}\text { Estudo } \\
\text { clínico }\end{array}$ & $\begin{array}{c}1094 \text { adolescentes } \\
\text { brasileiros } \\
(12-14 \text { anos) }\end{array}$ & $\begin{array}{c}\text { Hábitos parafuncionais e outras queixas de dor corporal } \\
\text { podem desempenhar um papel importante na presença de } \\
\text { dor em DTM em adolescentes. }\end{array}$ \\
\hline
\end{tabular}

Fonte: elaborado pelos autores.

Os sinais e sintomas de DTM normalmente descritos na literatura revisada para essa faixa etária estavam relacionados à sintomatologia dolorosa (dores articulares, de cabeça ou miofasciais), além de sons e estalidos; enquanto os hábitos deletérios e parafuncionais mais frequentemente relatados foram: mastigar bala/chicletes/pirulito, onicofagia, morder objetos/lábios/bochechas, apertamento diurno e bruxismo, mastigação unilateral, "play jaw", respiração bucal e sucção digital (FARSI, 2003; CAUÁS et al., 2004; FEITH RABAB, 2006; FRAGOSO et al., 2010; FRANCO-MICHELONI et al., 2015; KARIBE et al., 2015; MEJERSJÖ, OVESSON e MOSSBERG, 2015; OLIVEIRA et al., 2016; AGARWAL, SAHAH e SINHA, 2016; ANITHA e KUMAR, 2017; CHATZOPOULOS et al., 2017; BARRETO et al., 2018), que por estarem relacionados com a mastigação e outras funções, podem prejudicar a estabilidade neuromuscular do sistema estomatognático, e causar DTM. Outros fatores etiológicos não relacionados à hábitos parafuncionais como: fatores emocionais (relacionado ao nervosismo de concursos e vestibulares), estruturais, posturais (sentados por longo período de tempo de forma incorreta), além de dormir sempre do mesmo lado debruçado em cima das mãos ou apoiar mão sobre a face (MARPAUNG; LOBBEZOO e VAN SELMS, 2018), parecem exigir maior esforço muscular podendo aumentar a probabilidade de desenvolver sinais e sintomas de DTM (CAUÁS et al., 2004; PEREIRA et al., 2009).

Um exame detalhado da musculatura da mastigação, das ATMs e estruturas ligamentares podem revelar se os sintomas do paciente são de DTM, entretanto, a ausência de diretrizes específicas para o diagnóstico de DTM em crianças e adolescentes torna difícil o tratamento baseado em evidências. Apesar das dificuldades inerentes quanto à percepção e diagnóstico da DTM nessa faixa etária, o que requer avaliação detalhada e parâmetros específicos, a mesma deve ser tratada assim que detectada, para se evitar maior comprometimento na fase adulta (TOSATO e CARIA, 2006), sendo necessárias ações que visam a orientação para controle e eliminação destes hábitos na infância e adolescência (NILSON; LIST; DRANGSHOLT, 2007; HOWARD, 2013; ORTEGA; GUIMARÃES, 2013).

Neste contexto é fundamental alertar sobre a importância do diagnóstico e tratamento precoce das DTM na população jovem, uma vez que há alta prevalência nessa população e é um tema ainda pouco 
explorado na literatura. Conhecendo-se melhor seus fatores etiológicos, sinais e sintomas clínicos, tornase possível diagnóstico e instrução aos pacientes e responsáveis para evitar seu agravamento, fornecendo melhor prognóstico e mais qualidade de vida aos pacientes portadores de DTM.

\section{CONCLUSÃo}

Concluiu-se que na população jovem é frequente haver sinais e sintomas de DTM, sendo o gênero feminino o mais acometido; e que quanto maior a quantidade e duração destes sinais e sintomas de DTM, maior é a tendência de agravamento da severidade desta disfunção. Observou-se ainda, que hábitos parafuncionais como onicofagia, morder objetos/lábios/bochechas, mastigar bala/chicletes, apertamento dental e apoiar a mão sobre o queixo estavam frequentemente relacionados com a presença de DTM; e que outros fatores como estruturais, psicológicos, posturais e comportamentais também podem aumentar a probabilidade de desenvolver sinais e sintomas de DTM.

\section{REFERÊNCIAS}

AGARWAL, K.; SAHA, S.; SINHA, P. Prevalence of temporomandibular disorders and its association with parafunctional habits among senior-secondary school children of Lucknow, India. J Indian Assoc Public Health Dent. v.14, n.2, p.139-43, 2016.

ANITHA, KUMAR, V.J. Association between Harmful Oral Habits and Signs and Symptoms of TMD among Adolescents. Research Journal of Pharmacy and Technology. v.10, n.8, p.2668-70, Aug. 2017.

ARAUJO, L.G.; COELHO, P.R.; GUIMARÃES, J.P. Associação Entre os Hábitos Bucais Deletérios e as Desordens Temporomandibulares: Os Filhos Imitam os Pais na Adoção Destes Costumes? Pesq Bras Odontoped Clin Integr. v.11, n.3, p.363-69, 2011.

ARAVENA, P.C.; ARIAS, R.; ARAVENA-TORRES, R.; SEGUEL-GALDAMES, F. Prevalencia de trastornos temporomandibulares en adolescentes del Sur de Chile, 2015. Rev Clin Periodoncia Implantol Rehabil Oral. v.9, n.3, p.244-52, 2016.

AREBALO, I.R.; VEDOVELLO, S.A.S.; SANTAMARIA JUNIOR, M.; KURAMAE, M.; TUBEL, C.A.M. Relação entre disfunção temporomandibular e mordida cruzada posterior. RGO. v.58, n.3, p.323-6, 2010.

ATSÜ, S.S.; GÜNER, S.; PALULU, N.; BULUT, A.C.; KÜRKÇÜOĞLU, I. Oral parafunctions, personality traits, anxiety and their association with signs and symptoms of temporomandibular disorders in the adolescentes. AJOL. v.19, n.1, 2019.

BARBOSA, T.S.; MIYAKODA, L.S.; POCZTARUK, R.L.; ROCHA, C.P.; GAVIÃO, M.B.D. Temporomandibular disorders and bruxism in childhood and adolescence: review of the literature. Int J Pediatr Otorhinolaryngol. v.72, p.299-314, 2010.

BARRETO, K.; PACHÓN, C.; RODRÍGUEZ, L.; BARRIOS, M. Presence of Parafunctional Habits in Temporomandibular Articulation Disorders in University Students. Indian J Scien Tech. v.11, n.13, p.17, April 2018.

BENOLIEL, R.; ZINI, A.; ZAKUTO, A.; SLUTZKY, H. Subjective Sleep Quality in Temporomandibular 
Disorder Patients and Association with Disease Characteristics and Oral Health-Related Quality of Life. J Oral Facial Pain Headache. v.31, n.4, p.313-22, Fall 2017.

BERTOLI, F.M.P.; BRUZAMOLIN, C.D.; PIZZATTO, E.; LOSSO, E.M.; BRANCHER, J.A.; SOUZA, J.F; et al. Prevalence of diagnosed temporomandibular disorders: A cross-sectional study in Brazilian adolescents. PLoS One. v.13, n.2, p.e0192254, Feb.2018.

BONJARDIM, L.R.; GAVIÃO, M.B.D.; PEREIRA, L.J.; CASTELO, P.M. Anxiety and depression in adolescents and their relationship with signs and symptoms of temporomandibular disorders. Int J Prosthodont. 2005a;v.18, n.4, p. 347-53.

BRANCO, R.S.; BRANCO, C.S.; TESCH, R.S.; RAPOPORT, A. Freqüência de relatos de parafunções nos subgrupos diagnósticos de DTM de acordo com os critérios diagnósticos para pesquisa em disfunções temporomandibulares (RDC/TMD). R Dental Press OrtodonOrtop Facial. v.13, n.2, p.61-9, 2008.

CAMPI, L.B.; VISSCHER, C.M.; ONGARO, P.C.J.; BRAIDO, G.V.V.; FERNANDES, G.; GONÇALVES, D.A.G. Widespread Pain and Central Sensitization in Adolescents with Signs of Painful Temporomandibular Disorders. Journal of Oral \& Facial Pain \& Headache. v.34, n.1, p.83-91, 2020.

CARDOSO, L.M.; KRAYCHETE, D.C.; ARAÚJO, R.P.C. A relevância do apertamento dentário nas desordens temporomandibulares. Revista de Ciências Médicas e Biológicas. v.10, n.3, p.277-83, 2011.

CARLSSON, G.E.; EGERMARK, I.; MAGNUSSON, T. Predictors of signs and symptoms of temporomandibular disorders: a 20-year follow-up study from childhood to adulthood. Acta Odontol Scand. v.60, n.3, p.180-5, 2002.

CAUÁS, M.; ALVES, I.F.; TENÓRIO, K.H.C.; FILHO, J.B.; GUERRA, C.M.F. Incidências de Hábitos Parafuncionais e Posturais em Pacientes Portadores de Disfunção da Articulação Craniomandibular. Revista de Cirurgia e Traumatologia Buco-Maxilo-Facial. v.4, n.2, p.121-9, 2004.

CHATZOPOULOS, G.S.; SANCHEZ, M.; CISNEROS, A.; WOLFF, L.F. Prevalence of temporomandibular symptoms and parafunctional habits in a university dental clinic and association with gender, age, and missing teeth. Cranio. v.16, p.1-9, Nov. 2017.

DE MELO JR, P.C.; AROUCHA, J.M.C.N.L.; ARNAUD, M.; LIMA, M.G.S.; GOMES, S.G.F.; XIMENES, R.; et al. Prevalence of TMD and level of chronic pain in a group of Brazilian adolescents. PLoS ONE. v.14, n.2, p.e0205874, 2019.

EBRAHIMI, M.; DASHTI, H.; MEHRABKHANI, M.; ARGHAVANI, DANESHVAR-MOZAFARI, A. Temporomandibular Disorders and Related Factors in a Group of Iranian Adolescents: A Cross-sectional Survey. J Dent Res Dent Clin Dent Prospect. v.5, n.4, p.123-7, 2011.

FARSI, N.M.A. Symptoms and signs of temporomandibular disorders and oral parafunctions among Saudi children. Journal of Rehabilitation. v.30, p.1200-8, 2003.

FEITH RABAB, M. Signs and symptoms of temporomandibular disorders and oral parafunctions in urban 
Saudi Arabian adolescents: a research report. Head \& Face Medicine. v.2, n.25, 2006.

FERNANDES, G.; ARRUDA, M.A.; BIGAL, M.E.; CAMPARIS, C.M.; GONÇALVES, D.A.G. Painful Temporomandibular Disorder Is Associated With Migraine in Adolescents: A Case-Control Study. The Journal of Pain. 2019.

FERNANDES, G.; FRANCO, A.L.; SIQUEIRA, J.T.T.; GONÇALVES, D.A.G.; CAMPARIS, C.M. Sleep bruxism increases the risk for painful temporomandibular disorder, depression and non-specific physical symptoms. J Oral Rehabil. v.39, n.7, p.538-44, 2012.

FERNANDES, G.; FRANCO-MICHELONI, A.L.; SIQUEIRA, J.T.T.; GONÇALVES, D.A.G.; CAMPARIS, C.M. Parafunctional habits are associated cumulatively to painful temporomandibular disorders in adolescents. Braz. Oral Res. v.30, n.1, p.23, Feb. 2016.

FERNANDES, G.; VAN SELMS, M.K.A.; GONÇALVES, D.A.G.; LOBBEZOO, F.; CAMPARIS, C.M. Factors associated with temporomandibular disorders pain in adolescents. J Oral Rehabil. v.42, n.2, p.113-9, 2015.

FRAGOSO, Y.D.; ALVES, H.H.C.; GARCIA, S.O.; FINKELSZTEJN, A. Prevalence of parafunctional habits and temporomandibular dysfunction symptoms in patients attending a tertiary headache clinic. Arq Neuropsiquiatr. v.68, n.3, p.377-80, 2010.

FRANCO-MICHELONI AL, FERNANDES G, DE GODOI GONÇALVES DA, CAMPARIS CM. Temporomandibular Disorders in a Young Adolescent Brazilian Population: Epidemiologic Characterization and Associated Factors. J Oral Facial Pain Headache. v.29, n.3, p.242-9, Summer 2015.

GESCH, D.; BERNHARDT, O.; ALTE, D.; SCHWAHN, C.; KOCHER, T.; JOHN, U.; et al. Prevalence of signs and symptoms of temporomandibular disorders in an urban and rural German population: results of a population-based Study of Health in Pomerania. Quintessence Int. v.35, n.2, p.143-50, Feb.2004.

GONÇALVES, D.A.G.; DAL FABBRO, A.L.; CAMPOS, .J.A.D.B.; BIGAL, M.E.; SPECIALI, J.G. Symptoms of temporomandibular disorders in the population: an epidemiological study. J Orofac Pain. v.24, n.3, p.270-8, Jan. 2010.

GÖRÜŞ, Z.; ÜNER, D.D. Evaluation of the relation between TMJ disorders and oral habits on the 1st year students at a faculty of dentistry: A questionnaire study. Journal of Harran University Medical Faculty. v.16, n.2, p.202-206, 2019.

GOYATÁ, F.R.; TAIRA, N.V.; ALMEIDA, S.; SILVA, D.M.; TAIRA, C.V. Avaliação de sinais e sintomas de disfunção temporomandibular entre os acadêmicos do curso de Odontologia da Universidade Severino Sombra, Vassouras- RJ. Int J Dent. v.9, n.4, p.181-6, 2010.

HIRSCH, C.; HOFFMANN, J.; TÜRP, J.C. Are temporomandibular disorder symptoms and diagnoses associated with pubertal development in adolescents? An epidemiological study. J Orofac Orthop. v.73, n.1, p.6-8, 2012. 
HOWARD, J.A. Temporomandibular Joint disorders in children. Dent Clin North Am. v.57, n.1, p.99127, 2013.

KARIBE, H.; SHIMAZU, K.; OKAMOTO, A.; KAWAKAMI, T.; KATO, Y.; WARITA-NAOI, S. Prevalence and association of self-reported anxiety, pain, and oral parafunctional habits with temporomandibular disorders in Japanese children and adolescents: a cross-sectional survey. BMC Oral Health. v.15, n.8, 2015.

KHAN, K.; MULLER-BOLLA, M.; TEIXEIRA JUNIOR,O.A.; GORNITSKY, M.;GUIMARÃES, A.S.;VELLY, A.M. Comorbid conditions associated with painful temporomandibular disorders in adolescents from Brazil, Canada and France: A cross-sectional study. J Oral Rehabil. v.47, n.4, p.i-iv 417-424, April 2020.

KHAN, K.; GORNITSKY, M.; VELLY, A.M. Painful and non-painful comorbid conditions associated with painful TMD in adolescents from Montreal, Canada. [Master of Dental Sciences] Faculty of Dentistry McGill University Montreal, Quebec, Canada July 2016. 118p.

KHOJASTEPOUR, L.; VOJDANI, M.; FORGHANI, M. The association between condylar bone changes revealed in cone beam computed tomography and clinical dysfunction index in patients with or without temporomandibular joint disorders. Oral surgery, Oral medicine, Oral pathology and Oral radiology. v.123, n.5, p.600-5, 2017.

KIM, J.H.; RYU, J.W.; AHN, J.M. Relationship of Korean teenagers with temporomandibular disorders and sleeping hours. Oral Biology Research. v.39, n.1, p.47-54, 2015.

KITSOULIS, P.; MARINI, A.; TLIOU, K.; GALANI, V.; ZINPIS, A.; KANAVARES, P.; et al. Signs and Symptoms of Temporomandibular Joint Disorders Related To the Degree Of Mouth Opening and Hearing Loss. BMC Ear, Nose and Throat Disorders. v.11, n.5, 2011.

LOBBEZOO, F.; AHLBERG, J.; GLAROS, A.G.; KATO, T.; KOYANO, K.; LAVIGNE, G.J.; et al. Bruxism defined and graded: an international consensus. J Oral Rehabil. v.40, n.1, p.2-4, 2013.

MAIXNER, W.; DIATCHENKO, L.; DUBNER, R.; FILLINGIM, R.B.; GREENSPAN, J.D.; KNOTT, C.; et al. Orofacial pain prospective evaluation and risk assessment study - the OPPERA study. J Orofac Pain. v.12, n.11 Suppl, p.T4-11, 2011.

MARPAUNG, C.; LOBBEZOO, F.; VAN SELMS, M.K.A. Temporomandibular Disorders among Dutch Adolescents: Prevalence and Biological, Psychological, and Social Risk Indicators. Pain Research and Management. 17 Apr 2018, 2018:5053709.

MARPAUNG, C.; VAN SELMS, M.K.A.; LOBBEZOO, F. Prevalence and risk indicators of pain-related temporomandibular disorders among Indonesian children and adolescents. Community Dent Oral Epidemiol. v.46, n.4, p.400-406, Aug 2018.

MEJERSJÖ, C.; OVESSON, D.; MOSSBERG, B. Oral parafunctions, piercing and signs and symptoms of temporomandibular disorders in high school students. Acta Odontologica Scandinavica. v.74, n.4, p.279-284, 2015. 
Moraes et al.

MELCHIOR, M.O.; MAZZETTO, M.O.; FELÍCIO, C.M. Temporomandibular disorders and parafunctonal oral habits an anamnestic study. Dental Press J Orthod. v.17, n.2, p.83-7, 2012.

MOTGHARE, V.; KUMAR, J.; KAMATE, S.; KUSHWAHA, S.; ANAND, R.; GUPTA, N.; et al. Association Between Harmful Oral Habits and Sign and Symptoms of Temporomandibular Joint Disorders Among Adolescents. J Clin Diagn Res. v.9, n.8, p.ZC45-ZC48, Aug. 2015.

MOTTA, L.J.; GUEDES, C.C.; SANTIS, T.O.; FERNANDES, K.P.S.; MESQUITA-FERRARI, R.A.; BUSSADORI, S.K. Association Between Parafunction Habits and Signs and Symptoms of Temporomandibular Dysfunction Among Adolescents. Oral Health PrevDent. v.11, p.3-7, 2013.

NILSSON, I.M.; LIST, T. Does adolescent self-reported TMD pain persist into early adulthood? A longitudinal study. Acta Odontol Scand. v.78, n.5, p.377-383,2020.

NILSSON, I.M.; LIST, T.; DRANGSHOLT, M. Incidence and temporal patterns of temporomandibular disorder pain among swedish adolescentes. J Orofac Pain. v.21, p.127-32, 2007.

NILSSON, I.M.; LIST, T.; DRANGSHOLT, M. The reliability and validity of self-reported temporomandibular disorder pain in adolescents. J Orofac Pain. v.20, n.2, p.138-44, 2006.

NILSSON, I.M.; WILLMAN, A. Treatment Seeking and Self-Constructed Explanations of Pain an Pain Management Strategies Among Adolescents with Temporomandibular Disorder Pain. J Oral Facial Pain Headache. v.30, n.2, p.127-33, Spring 2016.

OHRBACH, R.; FILLINGIM, R.B.; MULKEY, F.; GONZALEZ, Y.; GORDON, S.; GREMILLION, H.; G., Slade. Clinical findings and pain symptoms as potential risk factors for chronic TMD: descriptive data and empirically identified domains from the OPPERA case-control study. J Pain. v.12, n.11 Suppl, p.T27-45, 2011.

OKESON, J.P. Tratamento das desordens temporomandibulares e oclusão. 7.ed. Rio de Janeiro: Elsevier, 2013. 512 .

OLIVEIRA, C.B.; LIMA, J.A.S.; SILVA, P.L.P.; FORTE, F.D.S.; BONAN, P.R.F.; BATISTA, A.U.D. Temporomandibular disorders and oral habits in high-school adolescents: a public health issue?, RGO. v.64, n.1, p.8-16, Jan./Mar. 2016.

ORAL, K.; KIIÇUK, B.B.; EBEOGLU, B.; DINÇER, S. Etiology of temporomandibular disorders pain. Agri. v.21, n.3, p.89-94, Jul. 2009.

ORTEGA, A.O.L.; GUIMARÃES, A.S. Fatores de risco para disfunção temporomandibular e dor orofacial na infância e na adolescência. Rev Assoc Paul Cir Dent. v.67, n.1, p.14-7, Jan./Mar 2013.

PEREIRA, L.J.; PEREIRA-CENCI, T.; CURY, A.A.D.B.; PEREIRA, S.M.; PEREIRA, A.C.; AMBROSANO, G.M.B.; GAVIÃO, M. B. D. Risk Indicators of Temporomandibular Disorder Incidences in Early Adolescence. Pediatric Dentistry. v.32, n.4, p.324-8, 2010. 
PEREIRA, L.J.; PEREIRA-CENCI, T.; PEREIRA, S.M.; CURY, A.A.D.B.; AMBROSANO, G.M.B.; PEREIRA, A.C.; GAVIÃO, M. B. D. Psychological factors and the incidence of temporomandibular disorders in early adolescence. Braz Oral Res. v.23, n.2, p.155-60, 2009.

PEREIRA-JÚNIOR, F.J.; FAVILLA, E.E.; DWORKIN, S.F. Critérios de diagnóstico para pesquisa das disfunções temporomandibulares (RDC/TMD). Tradução oficial para a língua portuguesa. Bras Clin Odontol Integr. v.8, n.47, p.384-95, 2004.

PERROTTA, S.; BUCCI, R.; SIMEON, V.; MARTINA, S.; MICHELOTTI, A.; VALLETTA, R. Prevalence of malocclusion, oral parafunctions and temporomandibular disorder-pain in Italian schoolchildren: An epidemiological study. J Oral Rehabil. v.46, n.7, p.611-616, July 2019.

REZENDE, M.C.R.; SOARES, B.M.S.; SILVA, J.S.; GOIATO, M.C.; TURCIO, K.H.L.; ZUIM, P.R.J.; ALVESCLARO, A. P. RFrequência de hábitos parafuncionais. Estudo Transversal em acadêmicos de Odontologia. Rev Odontol de Araç. V.30, n.1, p.59-62, 2009.

RIBEIRO-DASILVA, M.C.; FILLINGIM, R.B.; WALLET, S.M. Estrogen-Induced Monocytic Response Correlates with TMD Pain: A Case Control Study. Journal of Dental Research. v.96, n.3, p.285-91, 2017.

RUBIN, P.F.; EREZ, A.; PERETZ, B.; BIRENBOIM-WILENSKY, R.; WINOCUR, E. Prevalence of bruxism and temporomandibular disorders among orphans in southeast Uganda: A gender and age comparison. Cranio. v.30, p.1-7, May 2017.

SARIT, S.; RAJESH, G.; MITHUN, P.B.H.; SHENOY, R. Factors influencing the impact of temporomandibular disorders on oral health-related quality of life among school children age 12-15 years in Mangalore: An observational study. J Indian Assoc Public Health Dent. v.17, p.58-65, 2019.

SENA, M.F.; MESQUITA, K.S.F.; SANTOS, F.R.R.; SILVA, F.W.G.P.; SERRANO, K.V.D. Prevalência de disfunção temporomandibular em crianças e adolescentes. Rev Paul Pediatr. v.31, n.4, p.538-45, 2013.

SILVA, M.F;; VEDOVELLO, S.A.S.; VEDOVELLO FILHO, M.; VENEZIAN, G.C.; VALDRIGHI, H.C.; DEGAN, V.V. Temporomandibular disorders and quality of life among 12-year-old schoolchildren. Cranio. v.35, n.6, p.392-6, 2016.

ŠPALJ, S.; ŠLAJ, M.; ATHANASIOU, A.E.; ŽAK, I.; ŠIMUNOVIC, M.; ŠLAJ, M. Temporomandibular Disorders and Orthodontic Treatment Need in Orthodontically Untreated Children and Adolescents. Coll. Antropol. v.39, n.1, p.151-8, 2015.

STEIN, S.; HELLAK, A.; POPOVIC, N.; TOLL, D.; SCHAUSEIL, M.; BRAUN, A. Internal derangement in the temporomandibular joint of juveniles with clinical signs of TMD: MRI-assessed association with skeletal and dental classes. Journal of orofacial orthopedics = Fortschritte der Kieferorthopadie: Organ/ official journal Deutsche Gesellschaft fur Kieferorthopadie. v.78. n.1, p.32-40, 2017.

TANEJA, P.; NAGPAL, R.; MARYA, C.M.; M., KATARIA; S., SAHAY, V.; GOYAL, D. Temporomandibular Disorders among Adolescents of Haryana, India: A Cross-sectional Study. Int J Clin Pediatr Dent. v.12, n.6, p.500-506, 2019. 
TOSATO, J.P.; CARIA, P.H.F. Prevalência de DTM em diferentes faixas etárias. RGO. v.54, n.3, p.211-14, 2006.

VIEROLA, A.; SUOMINEN, A.L.; IKAVALKO, T.; LINTU, N.; LINDI, V.; LAKKA, H-M. Clinical signs of temporomandibular disorders and various pain conditions among children 6 to 8 years of age: the PANIC study. J Orofac Pain. v.26, n.1, p.17-25, 2012.

WAHLUND, K.; NILSSON, I.M.; LARSSON, B. Treating temporomandibular disorders in adolescents: a randomized, controlled, sequential comparison of relaxation training and occlusal appliance therapy. J Oral Facial Pain Headache. v.29, n.1, p.41-50, 2015. 